(C)2009 IEEE. Personal use of this material is permitted. However, permission to reprint/republish this material for advertising or promotional purposes or for creating new collective works for resale or redistribution to servers or lists, or to reuse any copyrighted component of this work in other works must be obtained from the IEEE. 


\title{
A Methodology for Transactional Risk Assessment and Decision Making in e- Business Interactions
}

\author{
Omar Hussain, Tharam Dillon, Elizabeth Chang and Farookh Hussain \\ Digital Ecosystems and Business Intelligence Institute, Curtin University of Technology \\ Curtin University of Technology, Perth, Australia \\ e-mail: o.hussain@cbs.curtin.edu.au
}

\begin{abstract}
The development and advancement of technologies have enabled users to complete their tasks efficiently. They have also provided them with various options and alternatives to complete and achieve their tasks. In such an environment, it is imperative for a user to make informed decisions that would ensure that its aims or objectives are achieved and its interaction experience is maximized. In the literature, various approaches for decision making have been proposed. But among those approaches, the notion of risk has been considerably ignored in the domain of e-business, despite its having been acknowledged as an important concept related to decision-making in any domain. In this paper, we propose a methodology by which users in an e-business domain can assess and analyze the level of transactional risk in the domain of e-business and then take it into consideration when making an informed interaction-based decision.
\end{abstract}

Keywords-e-business, informed decision, transactional risk, performance risk, financial risk.

\section{INTRODUCTION}

The growth of e-business activities is quite evident by considering the statistics of its adoption and utilization by the users in the recent past. The primary reason behind this is because of its capability to provide the users with enhanced functionality by which they can complete their tasks in much lesser time and with greater ease as compared to the previous interaction infrastructures. These functionalities keep on increasing with the constant development and advancement of e-business interaction infrastructures, thereby increasing the advantages to the users. The e-business paradigm can also be termed as a collaborative medium, where a user forms collaborations with other users in order to achieve its desired outcomes. Such architectures can be utilized to facilitate any type of business activity, the most popular being the e-marketplace where users buy or sell their goods to others users. But these architectures do not provide any guarantee to its users of always achieving their outcomes. So apart from all the advantages that this interaction infrastructure provides, interacting users have to constantly look out for those factors that will take its interaction towards a 'negative' outcome. Negative outcome signifies the non-achievement of desired outcomes by the interacting user and the experience of losses. So in order to enjoy the benefits of the e-business paradigm, users should make informed interaction-based decisions. We define informed interaction-based decisions as those whereby the user's interaction experience and benefits are maximized and any losses are minimized or alleviated. By making informed decisions, users can transform themselves to intelligent agents who can then act in a proactive way in order to achieve their goals.

Some notions or concepts that are important to consider while making an informed interaction-based decision are 'Security', 'Privacy', 'Trust' and 'Risk'. In the literature, various approaches to decision-making have been proposed in the area of e-business. But through analysis, we note that the notion of risk is not given its due importance during decision-making in such domains. Risk is a term with negative connotations used to represent (a) events that will result in experiencing a negative outcome in the interaction, and (b) the level and magnitude of possible loss that can be experienced in forming an interaction. Both these representations from risk play an important part during decision making, and are not determined by the analysis of trust, security and privacy in the collaboration. Subsequently, any decision that is being made without the analysis of risk does not take into consideration these important concepts; and hence, cannot be classified as being an informed one. In this paper, we highlight the importance of risk while decision-making in an e-business interaction and then propose an architecture which quantifies it and utilizes it while making an interaction-based decision in the ecommerce paradigm. The paper is organized as follows. In Section 2, we briefly distinguish between security, trust and risk and highlight the importance of risk while decisionmaking in an e-business interaction. In Section 3, we highlight the different sub-categories and characteristics according to which transactional risk in an interaction should be assessed. From Sections 4-7, we explain how transactional risk in an interaction can be quantified and then utilized for determining an interaction. In Section 8, we conclude the paper.

\section{Classification Between Security, Trust And} RISK WHILE DECIDING ON AN E-BUSINESS INTERACTION

Security is the act of taking appropriate measures by which the threats or the dangers are addressed, checked or eliminated. In an e-business interaction, security will help to analyze and keep the collaboration platform safe from the intrusion of outside disruptive forces. The notion of privacy will help to determine and keep the sensitive information of each user safe from un-authorized access. Trust will express the level to which a user can depend on another user to achieve its outcomes. Risk will express the consequences to the user as a result of the failure of its collaboration. 
Although each of these concepts is related to the same business interaction, the analysis which each provides is different and not interchangeable with any other. Further, the analysis of each of these concepts is important at different time periods of the collaboration for making an informed interaction-based decision. The notion of security and privacy are important in the initial phase of making an informed interaction-based decision, where the user from a group selects those users with whom to collaborate who satisfy its perceptions of interacting in a safe environment. But such analysis is not enough to make an informed interaction-based decision. To have a complete analysis, from the selected group, that user should be chosen who has the best capability to commit to the desired outcomes of the business interaction. This representation is achieved by the analysis of trust and risk in the business interaction. We consider this to be the later phase of the process of making an informed interaction-based decision.

A lot of work has been discussed in the literature for decision-making based on the assessment of security, privacy and trust in the interaction. But at the same time, the notion of risk has been ignored and not given its due importance. Risk analysis is important in influencing the behavior of the interaction initiating agent in the interaction because there is a substantial body of literature based on rational economics that argues that the decision to buy is based on the risk-adjusted cost-benefit analysis [1]. Thus, it commands a central role in any discussion related to a transaction. But regardless of its importance, it is incorrect to think that the term 'risk' can be defined globally or in a generic way which can then be applied to any discipline under discussion. The definition of the term 'risk' varies across disciplines according to how it is interpreted or how it best fits its object of analysis in the particular discussion. Taking this into consideration, it can be inferred that the way 'risk' is defined in one discipline may not be suited or utilized to define 'risk' in other disciplines, as their object of analysis might vary with the change of the discipline, thereby changing its interpretation and the sub-categories of its analysis. Even in a single discipline, there are various ways by which risk can be interpreted. For example, in the domain of e-business, the absence of adequate security mechanisms poses a risk to the successful completion of the business interaction. This is termed a security risk. Similarly, the non-preservation of the private information of the participants represents a threat to the successful unauthorized completion of the business interaction. This is termed a privacy risk. These types of risks are different from each other and will be addressed through an analysis of the concepts of security and privacy respectively. We consider these types of risks to be related to the initial part of the decision-making process in a business interaction. Another important representation of risk required in the later part of decision-making is the level to which the interacting user will not achieve its desired outcomes and the level of consequences that it will experience as a result of that in its business interaction. We term such representation of risk a transactional risk. This type of risk plays an important part during the later part of decision-making, while choosing a user with whom to interact. This risk is concerned with the actual achievement of desired outcomes which is different from the security and privacy risks and subsequently, it too should be considered while decision-making.

Although the importance of transactional risk while decision-making has been acknowledged by some researchers in the literature, while proposing their model they ignore it in favor of trust. In other words, they consider trust to be an authoritative concept in comparison with risk, and base their interaction-based decision on that. For example, Wang and Lin [2] state that trust represents the extent to which a party can depend on the other with relative assurance, even though 'negative consequences' are possible. But they do not propose an approach by which the negative consequences are quantified and considered while decision making. Aberer et al. [3] however, consider the possibility of agent ' $A$ ' cheating in the interaction, but they do not take into consideration the transactional risk while decision making, which gives the possible degree and magnitude of loss in interacting with agent ' $A$ ' due to the latter's cheating. From the approaches which ignore considering risk, Datta et al. [4] consider security as the key to any e-commerce infrastructure, and trust as one of its constituents of decision making. Damiani et al. $[5,6]$ propose an approach by which a peer chooses another peer with which to interact based on the level of trust. Hussain et al. [7] [8, 9] however, determine trust in the interaction according to its context-specific and dynamic property, but they do not consider the notion of transactional risk and possible loss when an agent decides to interact with another agent. Zheng et al. [10] consider the cost and utility function associated with an interaction while decision making, but they associate the cost with the 'rewards' of the consequences of decision. Akhter at al. [11] and Josang et al. [12] consider trust as the only factor in their approaches which motivates an agent in the interaction. Although Josang et al. [12] consider in their model the 'possible harm' and 'negative consequences', they do not consider transactional risk in their model as a factor to address this and utilize it in decision making. Hassell [13] proposes a method whereby an agent decides to trust another agent by considering the neurological and socialization characteristics of its brain apart from just considering the 'level of belief' expressed by trust, but they fail to consider the importance of transactional risk and its impact on an agent's brain while decision making. Similarly, Pearson et al. [14] propose an approach whereby a trust-based decision is made which omits to take transactional risk into consideration. But as mentioned earlier, no matter what technique is utilized for determining the level of trust in the interaction, ultimately they all represent the level of belief that an agent has in either the security aspects or in the other agent, or the degree of reliability to which an agent can depend on the other agent, or the credibility of an agent to perform a given task in the interaction, which is ascertained, named and expressed according to its interpretation by the researchers in their approaches. But the degree of trust in the interaction omits and does not represent the 'degree or magnitude of loss' in the interaction. 
With the evolving and ever developing technology of the modern age internet for facilitating e-commerce interactions, users would want to analyze the details of their interaction before taking an interacting-based decision. They would rather avoid deciding on an interaction from the onedimensional perspective analysis of the interaction, i.e. just based on the level of belief or trust that an agent has in interacting with the other agent; rather, along with it they would also want to take into consideration each possible outcome and the associated costs to them, while making an informed interaction-based decision. This is done by analyzing the level of failure along with the costs associated with those failures in the interaction, apart from just considering the level of belief in the interaction. This is within the scope of transactional risk in the interaction, which cannot be compensated for by analyzing the degree and level of trust. Hence, analyzing the level and degree of transactional risk in the interaction is very important when making an informed interaction-based decision, apart from considering just the level of trust. So in this paper, we will propose a comprehensive methodology by which a user initiating an interaction in an e-business interaction can ascertain beforehand the level of transactional risk in an interaction. Utilizing such assessments of risk while decision-making will lead to having a 'complete methodology' by which an informed interaction-based decision can be taken.

\section{Assessment Of Transactional Risk In E- BUSINESS INTERACTIONS}

We consider that a user initiating an interaction in an ebusiness domain wants to achieve certain desired outcomes, and in order to achieve those, wants to interact with another user who has the capability and who is willing to provide these outcomes to it. As is common in today's world, the other user in turn will receive a financial return from the interacting initiating user. We term the interaction initiating user, the risk assessing agent and the user with whom it has to decide whether to interact, the risk assessed agent. It is possible that there might a single risk assessed agent or a given set of risk assessed agents from which the risk assessing agent has to choose an agent with which to interact. Further there might be a different set of factors or functionalities which the risk assessing agent wants to achieve as its desired outcomes. We term each factor the assessment criterion of its interaction. We propose that the risk assessing agent should first communicate to the risk assessed agent its desired outcomes which it wants to achieve in interacting with it. Based on this, the two agents may negotiate with each other and the risk assessed agent may modify the required factors of the risk assessing agent according to its capability and willingness to provide them. If the risk assessing agent, based on the modified factors, concludes that it will achieve its objectives in the interaction, then it will form the 'expectations' of its interaction with the risk assessed agent. The expectations define how the users of the interaction are expected to behave so that the aim of the interaction is achieved. For example, let us consider that risk assessing agent ' $A$ ' wants to interact with a logistics company for transferring its goods. The context of the interaction is 'Transporting Goods' which we term ' $\mathrm{C}$ '. Let us assume that there are 5 different desired outcomes and the assessment criteria in its interaction are like (a) the goods should reach their destination undamaged (b) The goods should be delivered by $8 \mathrm{am}$ to the destination on the specified day (c) The logistics company should unpack the goods at the destination (d) The logistics company should provide a track and trace facility (e) The logistics company should complete the transaction for $\$ 8,000$. Based on the assessment criteria, the risk assessing agent enters into a negotiation phase with the logistics company. In the negotiation phase, the logistics company identifies those factors communicated by the risk assessing agent, which prevent it from fulfilling all the outcomes and meeting the assessment criteria, and subsequently modifies them according to how it promises to meet them and then communicates this back to the risk assessing agent 'A'. For example it may change the fifth assessment criterion which states that it will complete the transaction for $\$ 10,000$. If the risk assessing agent ' $A$ ' based on the modified factors from its assessment criteria concludes that it will achieve its objectives, then it can choose to interact with that logistics company for the transfer of its goods. This set of modified assessment criteria will form the 'expectations' in its future interaction with the logistics company. In other words, it is these factors that the risk assessing agent ' $A$ ' expects the logistics company to adhere to in order to provide it with what it desires in the interaction. Subsequently, the risk assessing agent ' $A$ ' while assessing the transactional risk in interacting with the logistics company should do it according to the expectations of its future interaction with it.

Transactional risk in the e-business domain should be determined according to specific sub-categories of performance risk and financial risk. Performance risk will represent the level of failure of the risk assessing agent in not achieving the expectations of its interaction with the risk assessed agent. Financial risk will represent the level of loss or consequences in the risk assessing agent not achieving its desired outcomes according to the expectations. Approaches have been proposed in the literature which define risk according to a uni-subcategory outcome of either just the probability of failure $[2,15-18]$ or as the outcomes or consequences of failure [19-21]. But none of them define risk by taking into consideration both these sub-categories of risk and subsequently, their analysis of risk is not according to its interpretation in the domain of e-business. Further, the specific characteristics according to which each sub-category of transactional risk has to be determined are context specific, assessment criteria specific and time specific. Context specific takes into consideration the specific purpose for which the interaction is being carried out. Assessment criteria specific takes into consideration the negotiated desired outcomes for which the interaction is being carried out. Time specific takes into consideration the dynamic nature of risk. All of these characteristics should be considered when analyzing the sub-categories of transactional risk in an e-business interaction. So the 
sequence of steps that we propose for the risk assessing agent to facilitate an informed interaction-based decision are:

1. The risk assessing agent should first form the expectations of its interaction with the risk assessed agent.

2. Determine the performance risk of the risk assessed agent in not committing to the formed expectations.

3. Determine the financial risk to the risk assessing agent as the result of failure in committing to its expectations.

4. Combine both the sub-categories to determine the numeric and linguistic level of transactional risk.

5. Determine the effect of its risk propensity or risk attitude on the determined level of transactional risk in deciding on an interaction.

In the next sections, we will present an overview of each of the abovementioned steps which will eventually lead the risk assessing agent to making an informed interactionbased decision.

\section{Assessment Of PerofmRnace Risk In E- BUSINESS INTERACTIONS}

As mentioned earlier, performance risk in the domain of e-business interaction represents the inability of the risk assessed agent to commit to the expectations of the interaction. In our previous work, we propose a comprehensive methodology by which the risk assessing agent can determine the performance risk of the risk assessed agent. For better understanding in the context of this paper, we give a brief overview of that in this section. To determine it according to the context specific, assessment criteria specific and dynamic characteristics of transactional risk, we proposed the Failure Scale and the term FailureLevel. The Failure Scale as shown in Figure 1 represents seven different levels of failure varying according to their severity, which could be possible in an interaction. We represent each level of failure on the Failure Scale which shows the corresponding severity of failure associated with it, as the 'FailureLevel' (FL). In other words, FailureLevel quantifies the level of failure according to the range of its severity on the Failure Scale. Each FailureLevel value on the Failure Scale quantifies and represents a different magnitude or severity of failure in the interaction.

\begin{tabular}{|c|c|c|}
\hline $\begin{array}{c}\text { Semantics of Failure } \\
\text { Level }\end{array}$ & $\begin{array}{c}\text { Probability of } \\
\text { Failure }\end{array}$ & FailureLevel \\
\hline Unknown & - & -1 \\
\hline Total Failure & $91-100 \%$ & 0 \\
\hline Extremely High & $71-90 \%$ & 1 \\
\hline Largely High & $51-70 \%$ & 2 \\
\hline High & $26-50 \%$ & 3 \\
\hline Significantly Low & $11-25 \%$ & 4 \\
\hline Extremely Low & $0-10 \%$ & 5 \\
\hline
\end{tabular}

Figure 1. The Failure Scale

It is possible that the performance risk which the risk assessing agent has to determine in interacting with the risk assessed agent might be based on a set of transactions with it over a given finite period of time, or it might be in a single transaction over a finite period of time. Furthermore, it is possible that the finite period of time of the risk assessing agent's interaction with the risk assessed agent may be limited to the current period of time, or might be a future point in time. If the interaction extends to a future point in time, then the risk assessing agent has to analyze the performance risk in interacting with the risk assessed agent over that future period of time. But as mentioned in the literature, transactional risk varies according to time and subsequently, it is imperative for the risk assessing agent to consider its dynamic nature while ascertaining its subcategories. To achieve this, we propose that the risk assessing agent:

1. Form the total time period of its interaction with the risk assessed agent and term it time space. The time space should be such that it takes into consideration the previous time periods which the risk assessing agent will utilize to study the risk assessed agent's incapability and then predict its performance risk in the future period of time.

2. Divide the time slots into different non-overlapping periods of time, termed time slots. The period of time at which the risk assessing agent starts its interaction with the risk assessed agent is termed the time spot.

3. The total time slots are further grouped into two categories, namely, the pre-interaction start time phase and post-interaction start time phase.

4. The pre-interaction start time phase refers to the period of time before the risk assessing agent starts its interaction with the risk assessed agent, whereas the post-interaction start time phase is the period of time after the risk assessing agent starts to interact with the risk assessed agent. The preinteraction start time phase spans the time from the beginning of the time space to the time spot of the interaction, whereas post-interaction start time phase is from the time spot of the interaction to the end of the time space.

5. To consider the dynamic nature of risk, we propose that the risk assessing agent, by utilizing either its past interaction experience or by soliciting recommendations, determine the FailureLevel of the risk assessed agent in each assessment criterion of its expectations.

6. If then combines the determined FailureLevel of each assessment criterion according to its significance in order to determine the FailureLevel of the risk assessed agent in each pre-interaction start time slot according to the expectations.

7. According to the determined FailureLevel in the preinteraction start time slots, the probability of occurrence of each level of failure on the Failure Scale is ascertained in each time slot of the post-interaction start time phase.

8. It is then combined to determine the FailureLevel Curve of the business interaction. The FailureLevel Curve quantifies and represents the different levels of performance risk of the risk assessed agent in committing to the expectations of the interaction.

The detail steps of the methodology by which the risk assessing agent can determine the sub-category performance risk of the risk assessed agent are mentioned in [22]. To extend the interaction scenario discussed in Section 3, let us consider that the risk assessing agent ' $\mathrm{A}$ ', by utilizing its past interaction history and recommendation from other users, determines the FailureLevel Curve (FLC) in forming an interaction with the logistics company as shown in 
Figure 2. This represents the different levels of failure along with their probability of occurrence of the risk assessed agent in committing to the expectations of the interaction.

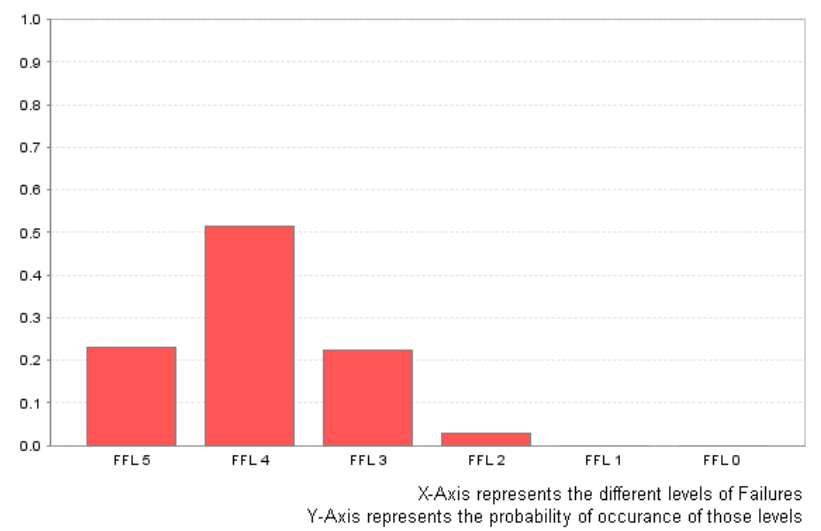

Figure 2. The FailureLevel Curve in agent 'A's interaction with the logistics company

Once the level of failure in the interaction has been determined, then the risk assessing agent should determine its impact on its financial resources at stake.

\section{Assessment OF FinANCIAL Risk In E-Business INTERACTIONS}

As with performance risk, it is possible that the risk assessing agent has to determine the financial risk in its interaction with a risk assessed agent that may be limited to the current period of time, or might extend to a future point in time. In such scenarios, an important point for the risk assessing agent to consider is the nature of investment of its resources decided in the expectations. For example, if there are 5 time slots in the risk assessing agent's ' $A$ ' interaction with the logistics company then there are three possibilities by which the agent ' $A$ ' can invest its resources. They are:

1. It invests the decided resources $(\$ 10,000)$ at the first time slot of the interaction,

2. It invests the decided resources in the last time slot of the interaction,

3. It invests its resources progressively in each time slot of the interaction.

In each case, the level of its resources at stake in the interaction varies, and subsequently, with it the levels of financial loss that it can experience will also vary. So it is important for the risk assessing agent to capture this variability of its resources at stake in the interaction before determining the financial risk. Further, there might be other uncertainties that are not dependent on the performance of the risk assessed agent, but they will still have an impact on the successful completion of the interaction in the resources formed in the expectations. All of these should be considered when determining the financial risk in the interaction. To achieve this, we propose that the risk assessing agent:

1. Determine the investments that it will make in each time slot of its interaction.

2. Plot the Amount Investment Curve (AIC) of its interaction. The Amount Investment Curve represents the accurate probability of an amount at stake in each time slot of the interaction from the net resources invested by the risk assessing agent.

3. Determine the Factual Amount Invested Curve (FAIC) of its interaction. The FAIC is the effect of the performance risk of the risk assessed agent on the AIC and it represents the required resources and the required probability of an amount from those resources to be kept at stake in the time period of its interaction.

4. Consider the non-dependent uncertainties that are specific to its business interaction (if any) and determine their impact on the FAIC to have the Total Factual Amount Invested Curve (TFAIC) of the interaction. The TFAIC represents the required resources with the probability to be kept at stake by considering the failure in dependent and uncertainties of non-dependent events.

5. Plot its maximum investment capacity (MIC) on the TFAIC to determine the Loss of Investment Probability (LOIP) and the Possible Consequences of Failure (PCF) in the business interaction.

The detailed steps by which the variability in the investments of resources is captured and then the effect of dependant and non-dependant uncertainties is determined on them are mentioned in $[23,24]$. To clarify, let us consider that there are 5 time slots in risk assessing agent 'A's interaction with the logistics company; and it invests its resources in each of those in the order of $\$ 2,000, \$ 3,000$, $\$ 1,000, \$ 2,000$ and $\$ 2,000$. Assuming that there are no nondependant events, then according to the performance risk of the logistics company the TFAIC of the interaction is as shown in Figure 4. Figure 4 shows the actual resources to be kept at stake along with their probability due to the uncertainties. Considering that the maximum investment capacity of the risk assessing agent is $\$ 3,000$ in each time slot, then the LOIP and the PCF are represented by the dotted line and shaded part respectively. Once the subcategories of transactional risk have been determined, then they should be combined to ascertain the level/s of transactional risk.

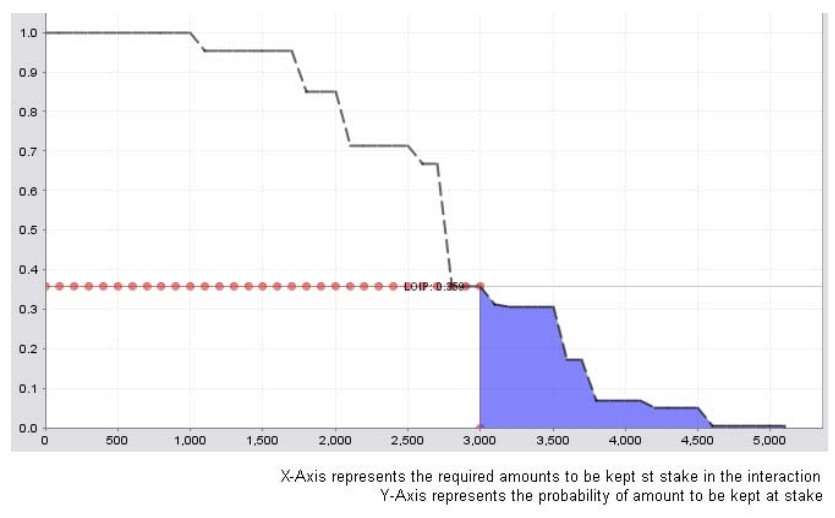

Figure 3. The Total Factual Amount Invested Curve (TFAIC) in agent 'A's interaction with the logistics company 


\section{DETERMINING THE TRANSACTIONAL RISK IN E- BUSINESS INTERACTIONS}

Once the performance risk and financial risk in an interaction has been determined, then the risk assessing agent should combine them to ascertain the transactional risk in the interaction. During this stage, it is reasonable to believe that there is uncertainty in the risk assessing agent's mind as the transactional risk is being determined in an interaction which is yet to be carried out; and that interaction will have a specific outcome but there is uncertainty as to exactly which outcome, of the likely ones, will occur. This uncertainty which is present in the risk assessing agent's mind can be classified into two types: 'ambiguity' and 'vagueness'. As our aim is to analyze the level of transactional risk, we define ambiguity as that uncertainty in the risk assessing agent's mind which represents its inability to identify the concrete level/s or magnitudes of transactional risk present in interacting with the other agent; whereas, vagueness is defined as that type of uncertainty which represents its inability to identify the degree or likelihood to which those levels will occur in the interaction. In order to eliminate these types of uncertainties from the risk assessing agent's considerations, we propose that it calculate the level and magnitude of transactional risk in interacting with a risk assessed agent by using two methods, namely the numerical approach and linguistic approach. The numerical approach utilizes possibility theory and determines the magnitudes or the levels of transactional risk in the interaction (on a scale of $0 . .100 \%$ ) along with the possibility of occurrence of those levels. The linguistic approach utilizes fuzzy sets and determines semantically the levels of transactional risk along with their likelihood of occurrence. The steps to determine the numeric and linguistic levels of performance risk are:

1. The risk assessing agent determines the focal elements and their degree of evidence of the inputs, PCF and LOIP in their universe of discourse.

2. The possibility of occurrence of each focal element of the inputs is determined and convolved to ascertain the transactional risk in the interaction.

3. The obtained representation of transactional risk represents the different levels or magnitudes of occurrence, on a scale of 0-100 along with the likelihood of occurrence of those levels. This shows the different levels of transactional risk that could be present along with the possibility or likelihood of occurrence of those levels.

4. To determine the linguistic levels of transactional risk, the possibility of occurrence of the fuzzy sets from the membership functions of each input is determined.

5. They are then fed into the inference engine, which based on fuzzy rules, computes the different fuzzy sets of transactional risk along with their degree of membership in the business interaction.

The detailed steps of determining the focal elements and the possibility of occurrence of the inputs and output are mentioned in $[25,26]$. Considering the performance risk and financial risk in the present interaction scenario of the risk assessing agent ' $\mathrm{A}$ ' with the logistics company, the different levels of numeric and linguistic transactional risk is as shown in Figures 4 and 5 respectively.



Figure 4. The possibility distribution of transactional risk in agent 'A's interaction with the logistics company

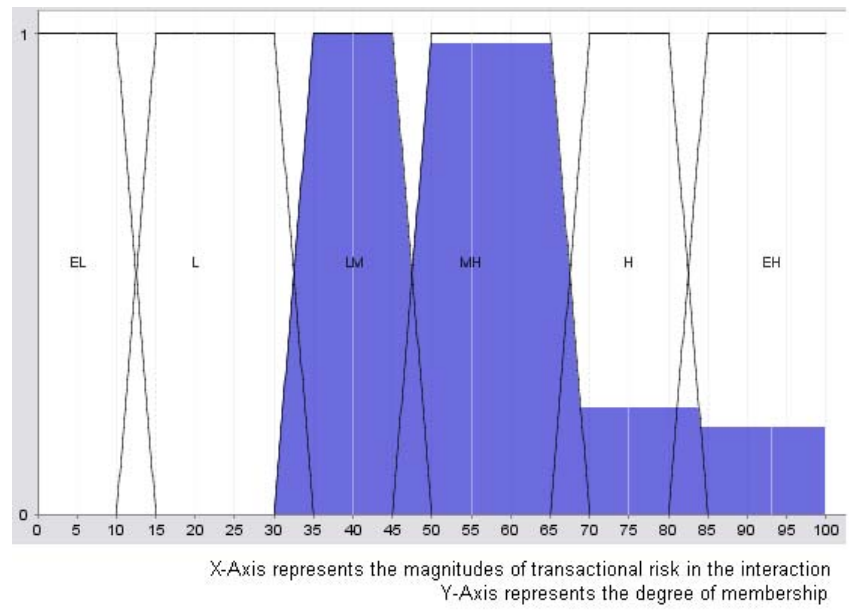

Figure 5. The linguistic levels of transactional risk in agent 'A's interaction with the logistics company

Once the sub-categories of transactional risk have been determined then they should be combined to ascertain the level/s of transactional risk.

\section{DECIDING ON AN INTERACTION BASED ON THE LEVEL OF TRANSACTIONAL RISK}

The decision making process of the risk assessing agent is dependent on its risk attitude or propensity. The risk attitude of the risk assessing agent determines how it 'sees' the level of transactional risk in interacting with the risk assessed agent, and based on this, which levels of transactional risk in the interaction are acceptable to it and which are not. It is important to note that no two risk assessing agents might have the same risk attitude, and with the variation in their risk attitudes, their approaches to decision making in the interaction vary. Also, the risk attitude of an agent might not be the same throughout. Subsequently, while making the decision, it is very important for the risk assessing agent to first ascertain its accurate risk propensity at that given period of time and 
then to determine its impact on the level of transactional risk in the interaction, for making an informed determination on an interaction. To achieve this, we propose:

1. Three levels for the risk attitude of the risk assessing agent, namely risk averse (RA), risk neutral (RN) and risk taking (RT). These attitude are in the order of $\mathrm{RA}<\mathrm{RN}<\mathrm{RT}$ in taking risk in an interaction.

2. It is possible that the risk propensity level of the risk assessing agent might not always be a crisp value which corresponds totally to a given level, but might overlap across the different possible levels. To take such scenarios into account, we propose using a fuzzy inference system to capture the fuzziness of the risk attitude of the risk assessing agent.

3. The fuzzy inference system, according to the fuzzy rules, determines the maximum level of acceptable transactional risk according to its risk attitude and then determines its effect on the level of transactional risk in the interaction.

4. The operation of the fuzzy inference system is divided into two parts, the learning part and the computation part. In the learning part, the fuzzy inference system 'learns' about the tendency of the risk assessing agent in taking risk in the interaction, and in the computation part, it utilizes the determined tendency of the risk assessing agent in computing the recommended risk-based decision in forming an interaction with the risk assessed agents.

5. The effect in the computation part is determined by two fuzzy sets, namely Proceed and Don't Proceed; which represents the two possibilities for the risk assessing agent to consider while decision making in an interaction. Further, the likelihood to which each output fuzzy set quantifies is determined. This will help the risk assessing agent to determine the levels at which transactional risk is acceptable. It can use this analysis further when it carries out the process of risk management.

6. The degree to which the output fuzzy sets 'Proceed' and 'Don't Proceed' quantifies in interacting with an agent, are according to the predicate/s of the transactional risk and its/their possibility of occurrence present in interacting with that agent, which is/are then further classified depending on whether it is/they are acceptable or unacceptable to the risk assessing agent according to its risk propensity or risk attitude.

The fuzzy inference system along with the fuzzy rules to capture the variability of the risk assessing agent's risk propensity and then determine its effect on an interaction are mentioned in [27]. Continuing the present example and considering the risk propensity of the risk assessing agent as a combination of Risk Neutral and Risk Taking with DOM 0.2 and 0.8 respectively; the strength to which the output fuzzy sets Proceed and Don't Proceed quantifies are 100\% and $0 \%$ respectively. This represents that all levels of determined transactional risk are acceptable to the risk assessing agent ' $\mathrm{A}$ ' according to its risk attitude, and it is possible to choose that logistics company with which to form an interaction.

So an overview of the methodology that we propose for considering the notion of risk while decision-making in ebusiness interaction is shown in Figure 6. The proposed

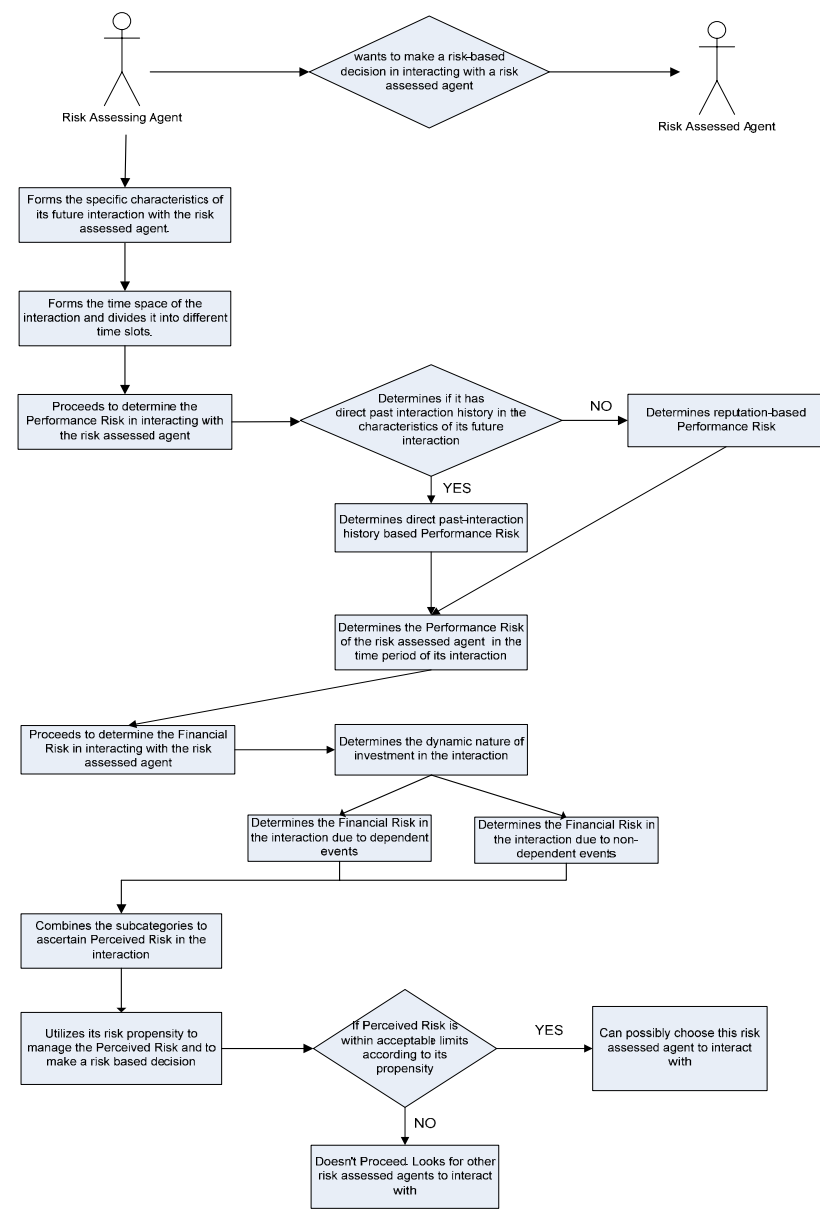

Figure 6. Overview of the risk-based decision making methodology

approach consists of a comprehensive set of steps by which an important problem of analyzing and considering transactional risk while decision-making e-business interactions is addressed in the literature. It captures the various dependent and non-dependent uncertainties that may produce a negative outcome for the interaction, determines their probability of occurrence and their impact on the successful completion of the interaction. It then determines whether or not the determined level of transactional risk is acceptable to the risk assessing agent according to its risk propensity. Such an approach introduces the notion of transactional risk when decision making and complements the various existing approaches to decision-making proposed in the literature.

\section{VIII.CONCLUSION}

In this paper, we proposed a methodology by which the interaction initiating user can analyze and consider the level of transactional risk while deciding on an e-business interaction. Our proposed approach takes into consideration the different sub-categories of transactional risk and determines it according to its context specific, assessment criteria specific and dynamic properties. Whilst the individual method of determining the different aspects of 
transactional risk were discussed earlier, this paper presents an integration of those different methods for solving subaspects of the overall problem of analyzing and considering transactional risk in an e-business interaction. By utilizing our approach and considering it with the proposed approaches in the literature to assess and analyze security, privacy and trust; an interaction initiating agent can make an informed interaction based decision in the e-business domain. Our proposed approach also determines the level to which the determined level of transactional risk is not acceptable to the risk assessing agent, according to its risk propensity. These levels of transactional risk must be considered when carrying out the process of risk management. This is our future work.

\section{REFERENCES}

[1] Sander Greenland, "Bounding Analysis as an Inadequately Specified Methodology," Risk Analysis, vol. 24, pp. 1071-1083, October 2004.

[2] Yan Wang, Duncan S. Wong, Kwei-Jay Lin, and V. Varadharajan, "Evaluating transaction trust and risk levels in peer-to-peer e-commerce environments," Information Systems and E-Business Management, vol. 6, pp. 25-48, January 2008.

[3] Karl Aberer and Z. Despotovic, "Managing trust in a Peer-2-Peer Information System," in Proceedings of the Tenth International Conference on Information and Knowledge Management (CIKM'01), Atlanta, Georgia, USA, 2001, pp. 310 - 317.

[4] Anwitaman Datta, Manfred Hauswirth, and K. Aberer, "Beyond "web of trust": Enabling P2P E-commerce," in IEEE International Conference on E-Commerce (CEC'03), Newport Beach, CA, USA, 2003, pp. 303-312.

[5] Ernesto Damiani, Sabrina De Capitani di Vimercati, Stefano Paraboschi, and Pierangela Samarati, "Managing and Sharing Servents' Reputations in P2P Systems," IEEE Transactions on Knowledge and Data Engineering, vol. 15, pp. 840-854, July/August 2003.

[6] Ernesto Damiani, Sabrina De Capitani di Vimercati, Stefano Paraboschi, Pierangela Samarati, and F. Violante, "A reputation-based approach for choosing reliable resources in peer-to-peer networks," in Proceedings of the 9th ACM conference on Computer and communications security Washington, DC, USA 2002, pp. $207-216$

[7] Farookh Khadeer Hussain, Elizabeth Chang, and T. S. Dillon, "Trustworthiness and CCCI metrics in P2P communication," Journal of Computer Systems: Science \& Engineering, vol. 19, pp. 173-190, 2004.

[8] Elizabeth Chang, Farookh Khadeer Hussain, and T. S. Dillon, "Trustworthiness Measure for e-Service," in Third Annual Conference on Privacy, Security and Trust (PST'05), New Brunswick, Canada, 2005.

[9] Elizabeth Chang, Tharam Dillon, and F. K. Hussain, Trust and Reputation for Service-Oriented Environments. West Sussex, England: John Wiley \& Sons, Ltd, 2006.

[10] Xiaoqing Zheng, Zhaohui Wu, Huajun Chen, and Y. Mao, "A Scalable Probabilistic Approach to Trust Evaluation," in Fourth International Conference on Trust Management (iTrust'06), Pisa, Italy, 2006, pp. 423-438.

[11] Fahim Akhter, Dave Hobbs, and Z. Maamar, "Determining the Factors which Engender Customer Trust in Business-to-Consumer (B2C) Electronic Commerce," in IEEE International Conference on E-Commerce Technology (CEC 2004), San Diego, CA, 2003.

[12] Audun Jøsang, Claudia Keser, and T. Dimitrakos, "Can We Manage Trust?," in Third International Conference on Trust Management (iTrust 2005), Rocquencourt, France, 2005, pp. 93-107.
[13] Lewis Hassell, "Affect and Trust," in Third International Conference on Trust Management (iTrust'05), France, 2005, pp. 131-145.

[14] Siani Pearson, Marco Casassa Mont, and S. Crane, "Persistent and Dynamic Trust: Analysis and the Related Impact of Trusted Platforms," in Third International Conference on Trust Management (iTrust'05), France, 2005, pp. 355-363.

[15] Li Xiong and L. Liu, "A Reputation-Based Trust Model for Peer-to-Peer eCommerce Communities," in International Conference on E-Commerce Technology (CEC'03), Newport Beach, California, USA 2003, pp. 275-284.

[16] Nathan Griffiths, "Task Delegation using Experience Based MultiDimensional Trust," in Proceedings of the 4th International Conference on Autonomous Agents and Multiagent Systems (AAMAS'05) Utrecht, The Netherlands, , 2005, pp. 489-496.

[17] Brian J. Corbitt, Theerasak Thanasankit, and Han Yi, "Trust and e-commerce: a study of consumer perceptions," Electronic Commerce Research and Applications, vol. 2, pp. 203-215, 2003.

[18] Cristiano Castelfranchi and R. Falcone, "Trust Is Much More than Subjective Probability: Mental Components and Sources of Trust," in Proceedings of the 33rd Hawaii International Conference on System Sciences, Hawaii, 2000, pp. 1-10.

[19] Iris Bohnet and Richard Zeckhauser, "Trust, risk and betrayal," Journal of Economic Behavior \& Organization, vol. 55, pp. 467-484, 2004.

[20] Gang Xie, Jin-long Zhang, and Kin Keung Lai, "Web-Based Risk Avoidance Group Decision Support System in Software Project Bidding " in International Conference on Web Intelligence and Intelligent Agent Technology Hong Kong, 2006, pp. 180-183.

[21] Colin English, Waleed Wagealla, Paddy Nixon, Sotirios Terzis, Helen Lowe, and A. McGettrick, "Trusting Collaboration in Global Computing Systems " in The First International Conference on Trust Management (iTrust'03), Heraklion, Crete, Greece, 2003, pp. 136-149.

[22] Omar K. Hussain, Elizabeth Chang, Farookh K. Hussain, and Tharam S. Dillon, "Determining the Failure Level for Risk Analysis in an e-commerce Interaction," in Advances in Web Semantics: A state-of-the Art Semantic Web, E. C. Tharam S. Dillon, Robert Meersman, and Katia Sycara, Ed.: Springer, 2008.

[23] Omar Hussain and Tharam Dillon, "Ascertaining the Financial Loss from Nondependable Events in Business Interactions by using the Monte Carlo method," in The Fourth International Conference on Availability, Reliability and Security (ARES'09), Fukuoka, Japan 2009, pp. 406-411.

[24] Omar Hussain, Tharam Dillon, Elizabeth Chang, and Farookh Hussain, "Determining the Net Financial Risk for Decision Making in Business Interactions," in 2009 International Conference on Advanced Information Networking and Applications, 2009, pp. 822-829.

[25] Omar K. Hussain, Elizabeth Chang, Tharam S. Dillon, and Farookh K. Hussain, "Ascertaining the Semantic and Linguistic Level of Perceived Risk in e-Business Interactions," in IEEE International Conference on e-Business Engineering (ICEBE'08), IEEE, Ed. Xian, China: IEEE, 2008, pp. 723-727.

[26] Omar Hussain, Elizabeth Chang, Tharam Dillon, and F. Hussain, "Determining the Level of Perceived Risk in e-business Web 2.0 Interactions " in 2008 IEEE International Conference on e-Business Engineering (ICEBE'08), Xian, 2008, pp. 1320.

[27] Omar Hussain, Tharam Dillon, and Elizabeth Chang, "A Fuzzy Inference Model for Risk based Informed Decision-Making in e-Business," in Proceedings of the 12th International Conference on Network-Based Information Systems (NBiS'09), Indianapolis, USA, 2009. 